\title{
Liquid Crystal Droplets Dispersed in Ferroelectric Copolymer of Vinylidene Fluoride and Trifluoroethylene
}

\author{
Tomonori KodA, Yoshihiro MoRITo, and Susumu IKEDA \\ Department of Polymer Science and Engineering, \\ Faculty of Engineering, Yamagata University, \\ 4-3-16 Jonan, Yonezawa 992-8510, Japan
}

(Received January 11, 2001; Accepted March 25, 2001)

\begin{abstract}
Microdroplets of antiferroelectric liquid crystal 4-(1-Methylheptyloxycarbonyl)phenyl 4'Octyloxybiphenyl-4-carboxylate(MHPOBC) were dispersed in ferroelectric matrix copolymer of vinylidene fluoride and trifluoroethylene $(\mathrm{P}(\mathrm{VDF} / \mathrm{TrFE}))$ by solvent-induced phase separation. We measured spontaneous polarization, dielectric constant and electro-optical properties of the present composite materials. Discussion is made on how the polarization structure of the ferroelectric matrix affects electro-optical properties of the materials. The polarization of the matrix polymer effectively helps the external field which induces optical switching of the materials.

KEY WORDS Ferroelectrics / Lorentz Field / Polymer Dispersed Liquid Crystals (PDLC) / Copolymer of Vinylidene Fluoride and Trifluoroethylene (P(VDF/TrFE)) / Liquid Crystal / Electro-Optical Property / 4-(1-Methylheptyloxycarbonyl)phenyl 4'-Octyloxybiphenyl-4-carboxylate (MHPOBC) /
\end{abstract}

Poly(vinylidene fluoride) (PVDF) and random copolymers of vinylidene fluoride and trifluoroethylene $(\mathrm{P}(\mathrm{VDF} / \mathrm{TrFE}))$ show the ferroelectric $\beta$ phase. ${ }^{1}$ The ferroelectricity stems from alignment of slightly deflected planar chain molecules in the crystalline structure. ${ }^{2,3}$ Free-surface annealing gives rise to a significant increase in the crystallinity of $\mathrm{P}(\mathrm{VDF} / \mathrm{TrFE})$ and this enhances the scope of application. ${ }^{4,5}$

One characteristic of the ferroelectricity is spontaneous polarization which can be switched by applied electric field. Our interest here is how the spontaneous polarization affects the electro-optical property of liquid crystals dispersed in $\mathrm{P}(\mathrm{VDF} / \mathrm{TrFE})$.

There are mainly two structures of phase separation for polymer/liquid-crystal composite materials. One is the polymer network type ${ }^{6,7}$ and the other is the microdroplet type which contains liquid crystals dispersed as microdroplets in polymer matrix. ${ }^{8,9}$ This paper studies the microdroplet type with $\mathrm{P}(\mathrm{VDF} / \mathrm{TrFE})$ as polymer matrix.

When we have a cavity in the polarized material, an electric field is induced by polarization charges on the surface of the cavity. It is called Lorentz field. ${ }^{11}$ The Lorentz field is originally considered to avoid infinite summation of atomic interaction for the calculation of the local electric field. Tsutsumi, Davis, and DeReggi used electrochromism of dyes to estimate the local electric field of the polarized $\mathrm{P}(\mathrm{VDF} / \mathrm{TrFE}){ }^{10}{ }^{10}$ The local electric field is the sum of the Lorentz field, external electric field, depolarization field and the field from atoms inside the cavity.

In the present study we made actual cavities which contain liquid crystals in polarized $\mathrm{P}(\mathrm{VDF} / \mathrm{TrFE})$ matrix, and detected the effects of the Lorentz field on theoptical property of liquid crystals.

\section{EXPERIMENTAL}

Matrix polymer was random copolymer $\mathrm{P}(\mathrm{VDF} / \mathrm{TrFE})$ purchased from Daikin Kogyo Ltd. Molar fraction of $\mathrm{VDF}$ is $78 \%$. $\mathrm{P}(\mathrm{VDF} / \mathrm{TrFE})$ has ferroelectric-paraelectric transition temperature $T_{\mathrm{c}}$ which depends on the molar fraction of VDF. ${ }^{1}$ We confirmed with differential scanning calorimetry (DSC) at temperature change rate $10^{\circ} \mathrm{C}$ per second that endothermic peaks of the present $\mathrm{P}(\mathrm{VDF} / \mathrm{TrFE})$ show $T_{\mathrm{c}}=126^{\circ} \mathrm{C}$ and melting temperature $T_{\mathrm{m}}=149^{\circ} \mathrm{C}$ for heating process.

Liquid crystal MHPOBC: 4-(1-methylheptyloxycarbonyl)phenyl $4^{\prime}$-octyloxybiphenyl-4-carboxylate was provided by Chisso Petrochemical Corporation. It is a typical antiferroelectric liquid crystal showing phase sequence, ${ }^{12}$ Crystal $\left(84^{\circ} \mathrm{C}\right)$ Smectic $(\mathrm{Sm})-\mathrm{C}_{\mathrm{A}}^{*}\left(118.4^{\circ} \mathrm{C}\right) \mathrm{Sm}$ $\mathrm{C}_{\gamma}^{*}\left(119.2^{\circ} \mathrm{C}\right) \quad \mathrm{Sm}-\mathrm{C}^{*}\left(120.9^{\circ} \mathrm{C}\right) \quad \mathrm{Sm}-\mathrm{C}_{\alpha}^{*}\left(122^{\circ} \mathrm{C}\right) \mathrm{Sm}-\mathrm{A}$ $\left(148^{\circ} \mathrm{C}\right)$ Isotropic.

We made composite films through solvent-induced phase separation. We dissolved MHPOBC and P(VDF/ TrFE) into solvent: $N, N$-dimethylformamide (DMF). The solution took a single phase without phase separation. The solution was put on a hot plate in a vacuum to remove DMF. The removal of DMF caused phase separation creating microdroplets of MHPOBC in the matrix polymer $\mathrm{P}(\mathrm{VDF} / \mathrm{TrFE})$. After that we annealed the composite film at $140^{\circ} \mathrm{C}$ to increase crystallinity of $\mathrm{P}(\mathrm{VDF} /$ TrFE).

We studied electric properties of the films by measurement of electric displacement $(D)$ induced by applied electric field $(E)$ and measurement of dielectric constant.

D-E hysteresis was measured with Sawyer-Tower circuit. Triangular wave electric field was applied at 0.05 $\mathrm{Hz}$ with amplitude $100 \mathrm{MVm}^{-1}$. The D-E experiment was done at room temperature.

Dielectric constant at $800 \mathrm{~Hz}$ was measured with experimental setting described in ref 13 . Amplitude of the 


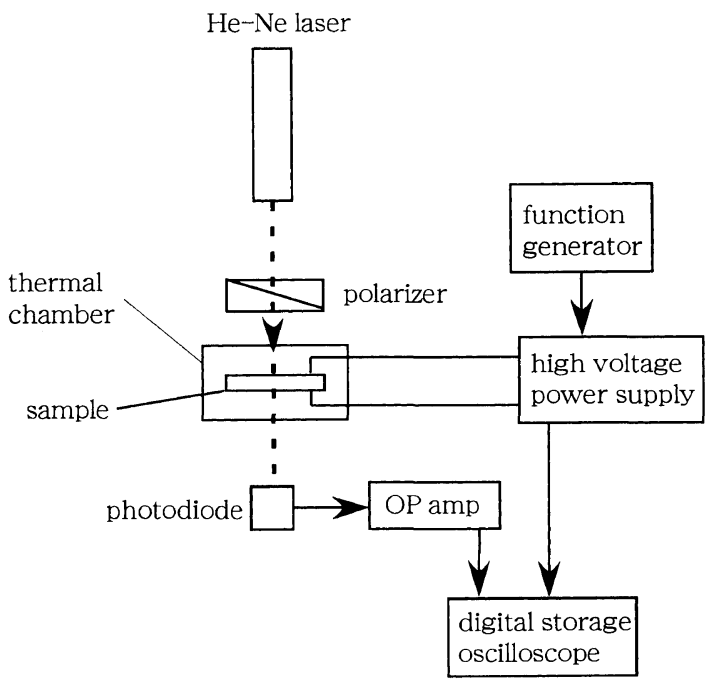

Figure 1. Schematic diagram of the system to measure electrooptical response.

input electric field was $3 \mathrm{MVm}^{-1}$. Temperature increase rate was $1^{\circ} \mathrm{C}$ per minute.

For D-E and dielectric constant measurements, gold electrodes were coated on both sides of the film. For dielectric constant measurement of pure MHPOBC, the sample was put between glass plates which had indium tin oxide (ITO) transparent electrodes.

Cross sections of the composite films were observed with scanning electron microscope (SEM) (JEOL Ltd. JSM-5310). To obtain the surfaces for SEM observation, pieces of epoxy resin containing sample films were broken under liquid nitrogen temperature and washed with ultra sonic vibration in methanol to remove MHPOBC on the surfaces. The surfaces were coated with gold to obtain better SEM images.

Optical response $(\mathrm{O})$ induced by $\mathrm{E}$ was measured by the experimental system described in Figure 1. Sample films for the O-E response measurement were put between ITO glass plates. To obtain better electric touch between the films and ITO electrodes, the temperature was increased before measurement up to $155^{\circ} \mathrm{C}$ which is higher than the melting temperature of $\mathrm{P}(\mathrm{VDF} / \mathrm{TrFE})$ and then increased from room temperature and kept at $90^{\circ} \mathrm{C}$ for the O-E experiments. At this condition MHPOBC takes antiferroelectric Sm-C ${ }_{\mathrm{A}}^{*}$ phase and the $\mathrm{P}(\mathrm{VDF} / \mathrm{TrFE})$ takes ferroelectric phase.

\section{RESULTS AND DISCUSSION}

\section{Electric Properties}

Figure 2 shows D-E hysteresis loops of the sample films at room temperature. At room temperature MHPOBC is in the crystal phase, while $\mathrm{P}(\mathrm{VDF} / \mathrm{TrFE})$ is in the ferroelectric phase. At high temperature where MHPOBC is in the liquid crystal phase, we could not orient the polarization of matrix $\mathrm{P}(\mathrm{VDF} / \mathrm{TrFE})$. Distribution of liquid crystal domains in the films and increase of conductivity at high temperature caused electric breakdown at a voltage that would induce the polarization reversal of $\mathrm{P}(\mathrm{VDF} / \mathrm{TrFE})$.

Polarization reversal in the crystal phase of MHPOBC has not been reported so far. Spontaneous polarization

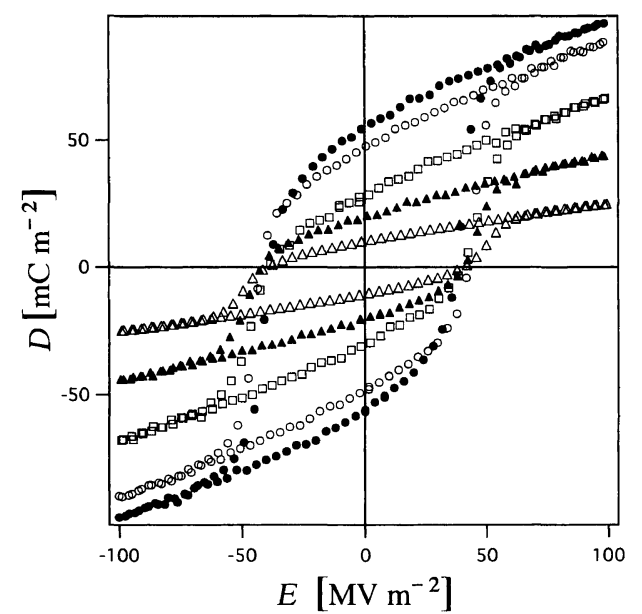

Figure 2. Plots of electric displacement $D$ vs. electric field $E$ of composite films at room temperature. Weight fractions of MHPOBC are 50\% (open triangles), 30\% (closed triangles), 20\% (open squares) and 10\% (open circles). Closed circles are for $\mathrm{P}(\mathrm{VDF} / \mathrm{TrFE})$ without liquid crystal.

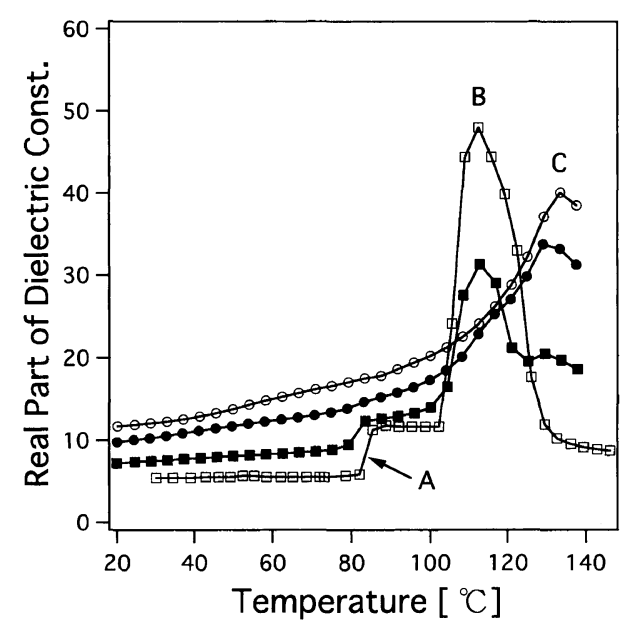

Figure 3. Dielectric constant at $800 \mathrm{~Hz}$ for heating process. Open circles: $\mathrm{P}(\mathrm{VDF} / \mathrm{TrFE})$. Closed circles: MHPOBC $10 \mathrm{wt} \%$. Closed squares: MHPOBC $50 \mathrm{wt} \%$. Open squares: MHPOBC. Jump $A$ is the transition from Crystal III to $\mathrm{Sm}-\mathrm{C}_{\mathrm{A}}^{*}$ phase of MHPOBC. ${ }^{12}$ Peak B corresponds to successive transitions from $\mathrm{Sm}-\mathrm{C}_{\mathrm{A}}^{*}$ to Sm-A phase ${ }^{16}$ and peak $\mathrm{C}$ ferroelectric-paraelectric transition of $\mathrm{P}(\mathrm{VDF} / \mathrm{TrFE})$.

$P_{\mathrm{s}}$ of the crystal of MHPOBC might be comparable to $1 \mathrm{mCm}^{-2}$ the typical $P_{\mathrm{s}}$ of MHPOBC in the liquid crystal phase. ${ }^{14,15}$ Comparing this value with the present $P_{\mathrm{s}}$ of $\mathrm{P}(\mathrm{VDF} / \mathrm{TrFE})$ at room temperature: $55 \mathrm{mCm}^{-2}$, we conclude that observed $P_{\mathrm{s}}$ arises from the matrix $\mathrm{P}(\mathrm{VDF} /$ TrFE).

Figure 3 shows the temperature dependence of dielectric constant. The real part of the dielectric constant of pure MHPOBC is quantitatively different from those obtained by Hiraoka et al. ${ }^{16}$ This is mainly due to the difference of applied voltage for measurement. We used the electric field of $6 \mathrm{MVm}^{-1}$ peak-to-peak magnitude, while they used $\sim 0.01 \mathrm{MVm}^{-1}$ peak-to-peak magnitude.

To see the morphology of the present materials, examples of SEM images are shown in Figures 4 and 5. They show cavities in which MHPOBC droplets were before removal. The shape of the microdroplet is not spherical 


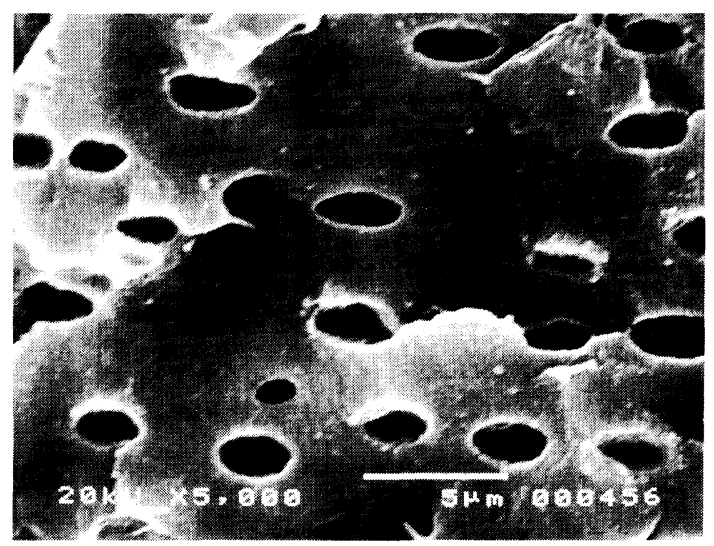

Figure 4. SEM images of cross sections of the composite film of $\mathrm{P}(\mathrm{VDF} / \mathrm{TrFE}) / \mathrm{MHPOBC}$. Weight fraction of MHPOBC is $10 \%$.

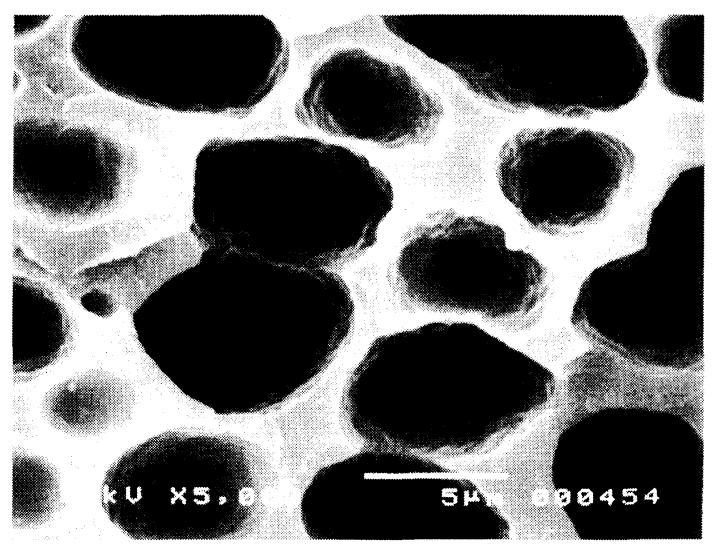

Figure 5. SEM images of cross sections of the composite film of $\mathrm{P}(\mathrm{VDF} / \mathrm{TrFE}) / \mathrm{MHPOBC}$. Weight fraction of MHPOBC is $40 \%$.

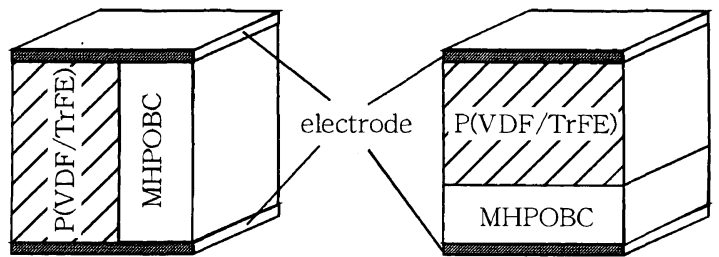

(a)

(b)

Figure 6. Parallel (a) and series connections (b) of $\mathrm{P}(\mathrm{VDF} / \mathrm{TrFE})$ and MHPOBC.

but ellipsoid.

One way to discuss the electric property of heterogeneous dielectrics is to use multilayer model. ${ }^{17}$ Here we consider the series and parallel models as described in Figure 6.

Denoting weight percentage of MHPOBC as $x$, dielectric constant $\varepsilon$ as a function of $x$ is, for the series model,

$$
\varepsilon(x)=\frac{\varepsilon_{\mathrm{p}} \varepsilon_{\mathrm{m}}\left[x \rho_{\mathrm{p}}+(100-x) \rho_{\mathrm{m}}\right]}{x \varepsilon_{\mathrm{p}} \rho_{\mathrm{p}}+(100-x) \varepsilon_{\mathrm{m}} \rho_{\mathrm{m}}}
$$

where $\varepsilon_{\mathrm{p}}$ is dielectric constant of $\mathrm{P}(\mathrm{VDF} / \mathrm{TrFE}), \varepsilon_{\mathrm{m}}$ is of MHPOBC, and $\rho_{\mathrm{p}}$ and $\rho_{\mathrm{m}}$ are mass density of $\mathrm{P}(\mathrm{VDF} /$

Polym. J., Vol. 33, No. 6, 2001

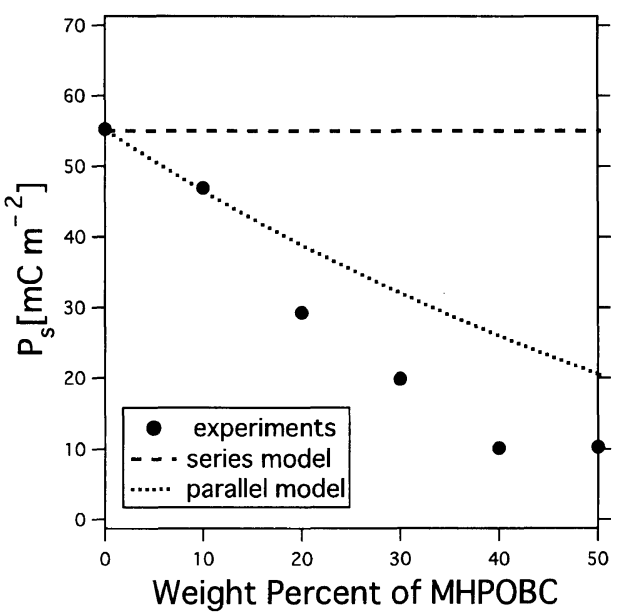

Figure 7. Dependence of spontaneous polarization on composition at room temperature.

TrFE) and MHPOBC, respectively. For the present calculation we used $\rho_{\mathrm{m}}=1.123 \mathrm{~g} \mathrm{~cm}^{-3}$ and $\rho_{\mathrm{p}}=1.88 \mathrm{~g} \mathrm{~cm}^{-3}$

For parallel model,

$$
\varepsilon(x)=\frac{x \varepsilon_{\mathrm{m}} \rho_{\mathrm{p}}}{(100-x) \rho_{\mathrm{m}}+x \rho_{\mathrm{p}}}+\frac{(100-x) \varepsilon_{\mathrm{p}} \rho_{\mathrm{m}}}{(100-x) \rho_{\mathrm{m}}+x \rho_{\mathrm{p}}}
$$

$P_{\mathrm{s}}$ and $E_{\mathrm{c}}$ as a function of $x$ are given for series model as

$$
\begin{gathered}
P_{\mathrm{s}}(x)=P_{\mathrm{s}}(0) \\
E_{\mathrm{c}}(x)=\frac{x \varepsilon_{\mathrm{p}} \rho_{\mathrm{p}}+(100-x) \varepsilon_{\mathrm{m}} \rho_{\mathrm{m}}}{x \varepsilon_{\mathrm{m}} \rho_{\mathrm{p}}+(100-x) \varepsilon_{\mathrm{m}} \rho_{\mathrm{m}}} E_{\mathrm{c}}(0)
\end{gathered}
$$

and for parallel model,

$$
\begin{gathered}
P_{\mathrm{s}}(x)=\frac{(100-x) \rho_{\mathrm{m}}}{(100-x) \rho_{\mathrm{m}}+x \rho_{\mathrm{p}}} P_{\mathrm{s}}(0) \\
E_{\mathrm{c}}(x)=E_{\mathrm{c}}(0)
\end{gathered}
$$

where we neglect the spontaneous polarization of MHPOBC. $P_{\mathrm{s}}$ of the series model is constant due to the continuity of electric flux between layers.

We compare our present results with the parallel and series models in Figures 7-9. Slightly increasing $E_{\mathrm{c}}$ and decreasing $P_{\mathrm{s}}$ show that our results are similar to the parallel model. However the parallel model does not agree with the dielectric constant of Figure 9, and our SEM images show that our materials are far from series or parallel cases. We have to consider the effects of conductive charges, refraction of electric field on surfaces, non-linearity of dielectric constant in a strong field, and so forth for further discussions.

\section{Electro-Optical Response of Non-Poled Films}

Measurements were done before electric poling of $\mathrm{P}(\mathrm{VDF} / \mathrm{TrFE})$ matrix. Our experimental set in Figure 1 detects the intensity of transmitted light. When we consider that the intensity of light in the sample is reduced 


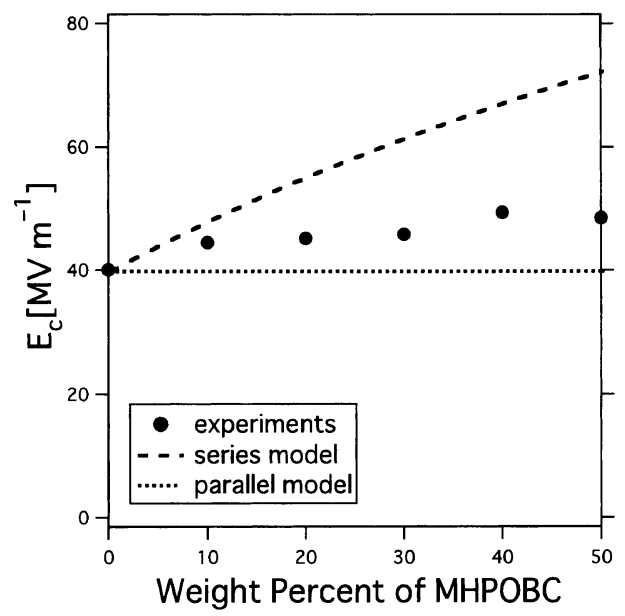

Figure 8. Dependence of coercive field on composition at room temperature.

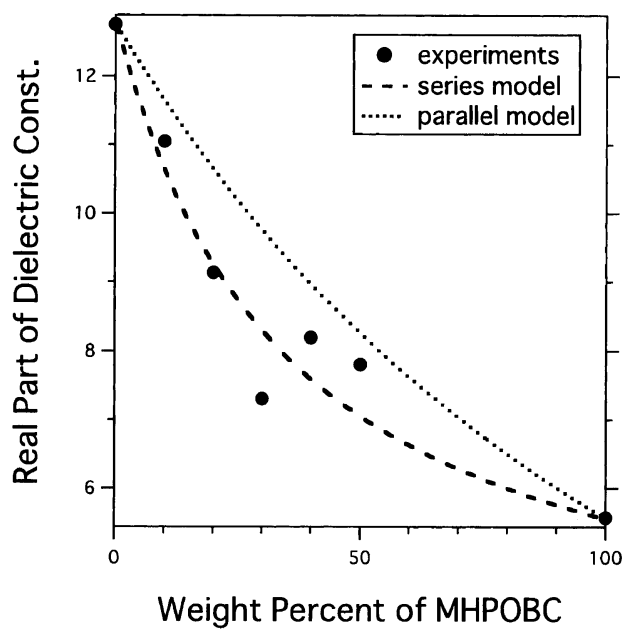

Figure 9. Dependence of dielectric constant on composition at $40^{\circ} \mathrm{C}$.

due to the Lambert's law, the intensity of incident light $I_{0}$ and transmitted light $I$ follows:

$$
I=c I_{0} \exp (-\alpha d)
$$

where $d$ is the film thickness, $\alpha(>0)$ constant which characterizes the scattering property of the sample and $c$ constant which describes decrease due to reflection on surfaces and adsorption in glasses.

Figure 10 shows change in $I$ for the triangular electric field at $90^{\circ} \mathrm{C}$. The shape of the hysteresis of $I$ changes depending on polarizing direction of incident light. This indicates anisotropy of the alignment of liquid crystals in a direction along the film surface. Though we intended no such anisotropy, we expect that this was given during solvent evaporation resulting from evaporation induced flow or slight temperature gradient.

Two peaks 1(1') and 2(2') in Figure 10 arise from twostep switching among tri-stable states of MHPOBC. ${ }^{15}$

After we adjusted the polarizing direction of incident light to obtain symmetric electro-optical hysteresis as Figure $10 \mathrm{~b}$, we applied a square electric field at $1 \mathrm{~Hz}$ to obtain the difference between electric field OFF state

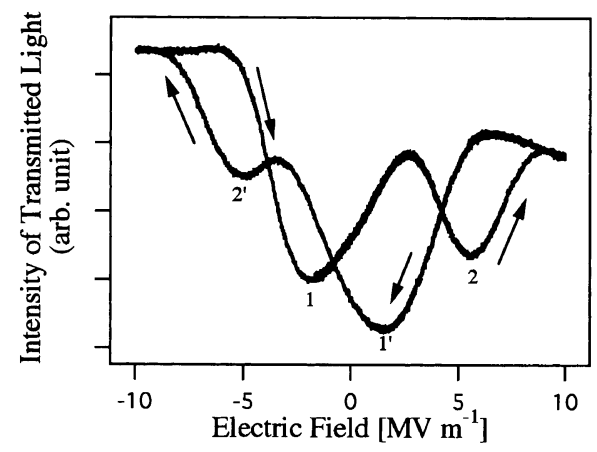

(a)

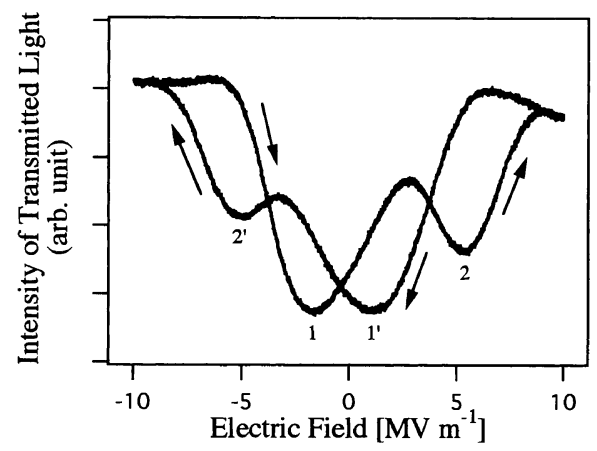

(b)

Figure 10. Relation between $I$ and electric field $E$ at $90^{\circ} \mathrm{C}$ for applied triangular electric field of frequency $100 \mathrm{~Hz}$ with amplitude $10 \mathrm{MVm}^{-1}$. (a) Before adjustment of polarization of incident light. (b) After adjustment of polarization of incident light, hysteresis is almost symmetric between $E$ and $-E$.

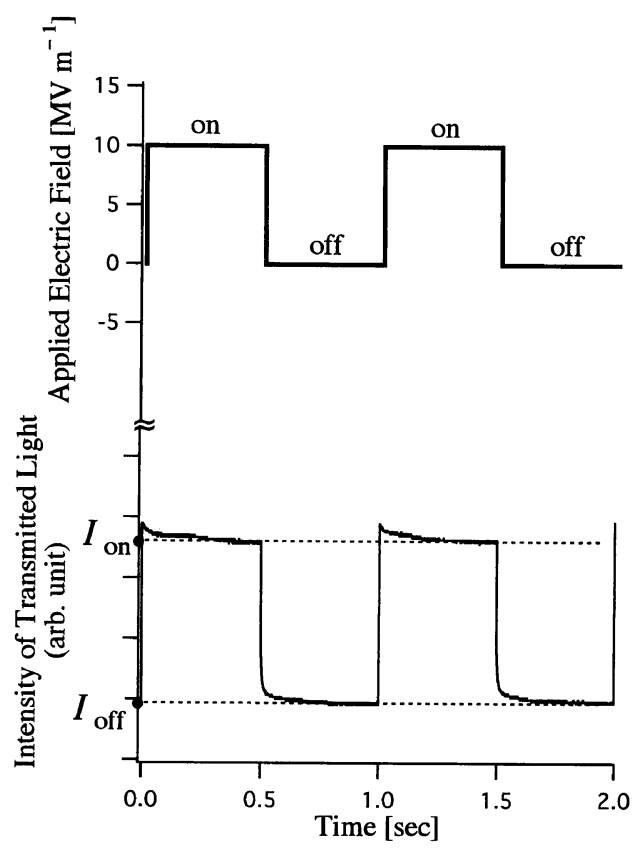

Figure 11. Response of $I$ to square electric field of $1 \mathrm{~Hz}$ at $90^{\circ} \mathrm{C}$. Height of the square electric field is $10 \mathrm{MVm}^{-1}$ in this case.

and ON state. Typical relation between applied square electric field and resulting change in $I$ is shown in Figure 11. In Figure 11, we denote the intensity of transmitted light at ON state as $I_{\text {on }}$, and OFF state as $I_{\text {off. }}$. 


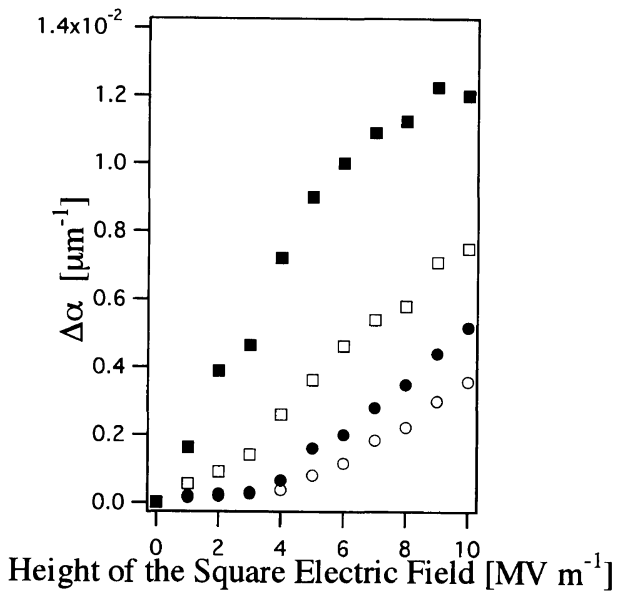

Figure 12. Dependence of $\Delta \alpha$ on height of square electric field for nonpoled films. Weight fractions of MHPOBC are $10 \mathrm{wt} \%$ (open circles), $20 \mathrm{wt} \%$ (closed circles), $30 \mathrm{wt} \%$ (open squares) and $50 \mathrm{wt} \%$ (closed squares).

Knowing eq 7, we denote the $\alpha$ at ON state as $\alpha_{\text {on }}$ and

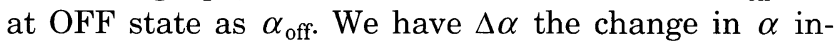
duced by the electric field as

$$
\Delta \alpha \equiv \alpha_{\text {off }}-\alpha_{\text {on }}=\frac{1}{d} \ln \left(I_{\text {on }} / I_{\text {off }}\right)
$$

$\Delta \alpha$ depends on the height of the square electric field. Figure 12 shows its dependence for various weight fraction of MHPOBC.

\section{Electro-Optical Property of the Poled Film}

We characterize the electro-optical property of films with $\Delta \alpha$ of eq 8 . Polarization structure of the matrix polymer was studied for effects on $\Delta \alpha$ of the material for a sample of $20 \%$ weight fraction of MHPOBC.

Non-poled films have polymer matrix which consists of polydomains of ferroelectric crystals. Polarizations in the matrix polymer are randomly oriented as indicated in Figure 13(A).

The polarization structure of the matrix can be changed at the point at which we turn off the applied electric field on the D-E hysteresis loop. Figure 14 shows a typical D-E hysteresis. When we turn off the electric field at the point $B$ of the hysteresis of Figure 14, we get the polarization structure in Figure 13(B). Polarizations in the film align parallel or antiparallel to the direction of electric field. Macroscopic spontaneous polarization of the film is almost zero in this case. At the point $\mathrm{C}$ of Figure 14 we get the structure in Figure 13(C). All polarizations are aligned to one direction. For the film of $20 \mathrm{wt} \%$ MHPOBC we measured the electric field dependence of $\Delta \alpha$ of these two polarization conditions, $\mathrm{B}$ and $\mathrm{C}$, in Figure 14. Measurement of $\Delta \alpha$ was done at $90^{\circ} \mathrm{C}$ and the $\mathrm{D}$ $\mathrm{E}$ hysteresis procedure was performed at room temperature. Figure 15 shows the results compared with nonpoled film. $\Delta \alpha$ changes after application of high electric field to polarize the materials. Applied electric field sometimes induces memory effect, ${ }^{18}$ that is, applied electric field changes optical property of OFF state.

When we have a spherical cavity filled with material

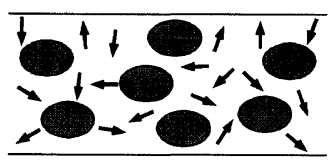

(A)

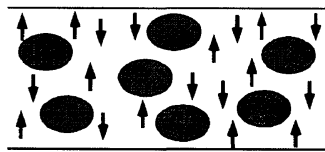

(B)

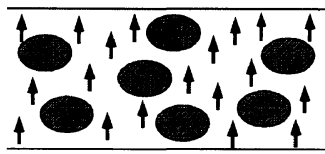

(C)

Figure 13. Schematic description of polarization structures. Arrows are polarization directions in the polymer matrix. Regions shaded contain liquid crystals. (A) Polarization directions in polymer matrix are randomly oriented. (B) Polarizations are aligned parallel or antiparallel to the direction perpendicular to film surface. (C) Polarization structure aligned to one direction.

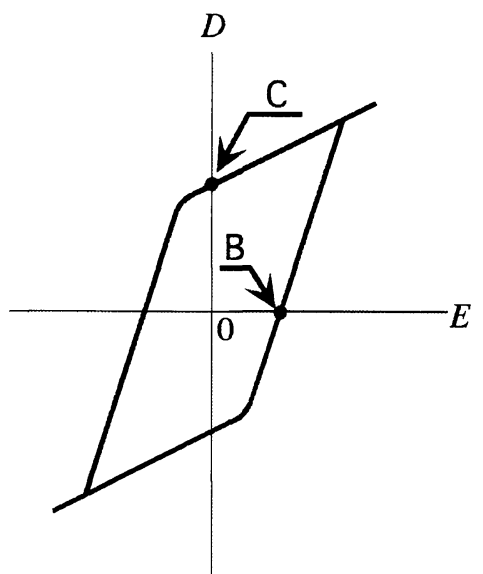

Figure 14. Schematic shape of D-E hysteresis.

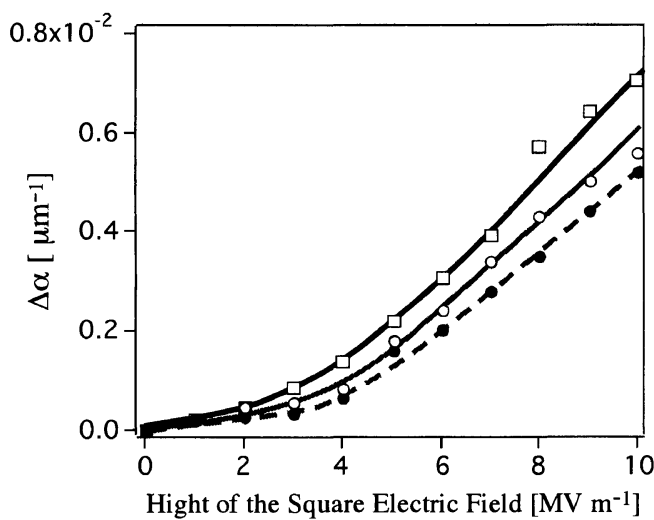

Figure 15. Electric field dependence of $\Delta \alpha$ for matrix polarizations in Figure 13. (a) Closed circles are for randomly oriented polarization (Figure 13(A)). (b) Open circles are for coexisting parallel and antiparallel polarization (Figure 13(B)). (c) Open squares are for polarization aligned in one direction (Figure 13(C)). Weight fraction of MHPOBC was $20 \%$. Measurement was done at $90^{\circ} \mathrm{C}$. Thick solid, thin solid, and thin dashed lines help visualize the plots (a), (b), and (c), respectively. 
of dielectric constant $\varepsilon$ in a continuum ferroelectric matrix of spontaneous polarization $P_{\mathrm{s}}$, the Lorentz field modified by the dielectric constant of the filler is given by $E_{\mathrm{s}}=P_{\mathrm{s}} /\left(3 \varepsilon_{0} \varepsilon\right)$, where $\varepsilon_{0}$ is the actual dielectric constant of vacuum.

The poling procedure changes not only $P_{\mathrm{s}}$ but also alignment of the symmetry axis of refractive index tensor of matrix polymer. The induced field and change in refractive index matching of liquid crystal with matrix polymer affect $\Delta \alpha$. To consider the pure effects of polarization induced field, we have to compare $\Delta \alpha$ of polarization structures $\mathrm{B}$ and $\mathrm{C}$ of Figure 13. The alignment of refractive index tensor does not change for these two polarization structures.

Denoting $\Delta \alpha$ of the polarization structure B and C, respectively, as $\Delta \alpha_{\mathrm{B}}$ and $\Delta \alpha_{\mathrm{C}}$, we write

$$
\Delta \alpha_{\mathrm{B}}=f(E),
$$

where $E$ is the external electric field and $f$ is a function which relates $E$ to $\Delta \alpha_{\mathrm{B}}$. If the polarization induced field $E_{\mathrm{s}}$ is simply added to $E, \Delta \alpha_{\mathrm{C}}$ should be

$$
\Delta \alpha_{\mathrm{C}}=f\left(E+E_{\mathrm{s}}\right)
$$

The above two equations mean that $\Delta \alpha_{\mathrm{C}}$ shifts from $\Delta \alpha_{\mathrm{B}}$ along the axis of the electric field by the magnitude of the polarization induced electric field.

From the difference between $\Delta \alpha_{\mathrm{B}}$ (open circles) and $\Delta \alpha_{\mathrm{C}}$ (open squares) of Figure 15, we estimated typical shift to be $E_{\mathrm{s}} \sim 0.5 \mathrm{MVm}^{-1}$ for the present case.

For $\mathrm{P}(\mathrm{VDF} / \mathrm{TrFE})$ with $P_{\mathrm{s}}=55 \mathrm{mCm}^{-2}$, the Lorentz field in the spherical liquid crystal region of dielectric constant $\varepsilon=5.6$ is theoretically $E_{\mathrm{s}}=370 \mathrm{MVm}^{-1}$, which is much higher than the above value. We expect that screening by conductive charges or distributions of charges on electrodes easily reduces the field to the observed value.

\section{CONCLUSION}

This paper discusses the effects of the Lorentz field induced in the cavity in the ferroelectric $\mathrm{P}(\mathrm{VDF} / \mathrm{TrFE})$.

The Lorentz field is originally the contribution of the electrostatic interaction outside a fictitious cavity considered to avoid infinite summation of interatomic interaction. For liquid crystals dispersed in the ferroelectric matrix $\mathrm{P}(\mathrm{VDF} / \mathrm{TrFE})$, we may consider an actual
Lorentz field that comes from surface polarization charge of $\mathrm{P}(\mathrm{VDF} / \mathrm{TrFE})$ at the boundaries in the phase separated structure.

Though the present preliminary results do not show the pure effects of the Lorentz field, we could show that polarization structure of the matrix polymer affects the electro-optical properties of the material. As Figure 15 shows, poling procedure of the matrix polarization effectively gave rise to a bias for the electric field.

Acknowledgment. The authors thank Chisso Petrochemical Corporation for providing MHPOBC. This work was supported by the Japan Securities Scholarship Foundation and by a Grant-in-Aid from the Ministry of Education, Culture, Sports, Science, and Technology of Japan, No. 12650875.

\section{REFERENCES}

1. T. Furukawa, Phase Transitions, 18, 143 (1989).

2. R. Hasegawa, M. Kobayashi, and H. Tadokoro, Polym. J., 3, 591 (1972).

3. K. Tashiro, K. Takano, M. Kobayashi, Y. Chatani, and H. Tadokoro, Polymer, 25, 195 (1984).

4. H. Ohigashi, S. Akama, and K. Koga, Jpn. J. Appl. Phys., 27, 2144 (1988).

5. H. Ohigashi, K. Omote, and T. Gomyo, Appl. Phys. Lett., 66, 3281 (1995).

6. T. Kajiyama, Y. Nagata, E. Maemura, and M. Takayanagi, Chem. Lett., 679 (1979).

7. E. Shimada and T. Uchida, Jpn. J. Appl. Phys., Part 2, 31, L 352 (1992)

8. P. S. Drzaic, J. Appl. Phys., 60, 2142 (1986).

9. S. Zumer and J. W. Doane, Phys. Rev. A, 34, 3373 (1986).

10. N. Tsutsumi, G. T. Davis, and A. S. DeReggi, Macromolecules, 24, 6392 (1991).

11. C. Kittel, "Introduction to Solid State Physics," 6th ed, John Wiley \& Sons, Inc., New York, N.Y., 1986, pp 366-372.

12. K. H. Kim, Y. Takanishi, K. Ishikawa, H. Takezoe, and A. Fukuda, Liq. Cryst., 16, 185 (1994).

13. S. Ikeda, H. Suzuki, and S. Nagami, Jpn. J. Appl. Phys., Part 1, 31, 1112 (1992).

14. A. D. L. Chandani, Y. Ouchi, H. Takezoe, and A. Fukuda, Jpn. J. Appl. Phys., 27, L 276 (1988).

15. A. D. L. Chandani, T. Hagiwara, Y. Suzuki, Y. Ouchi, H Takezoe, and A. Fukuda, Jpn. J. Appl. Phys., 27, L729 (1988).

16. K. Hiraoka, A. Taguchi, Y. Ouchi, H. Takezoe, and A Fukuda, Jpn. J. Appl. Phys., 29, L103 (1990).

17. A. Miyamoto, H. Kikuchi, S. Kobayashi, Y. Morimura, and T. Kajiyama, Macromolecules, 24, 3915 (1991).

18. R. Yamaguchi and S. Sato, Jpn. J. Appl. Phys., Part 2, 31, L 254 (1992). 